\title{
Differences of thought
}

\author{
Dr. Thiyam Kiran Singh*, Ajaz Ahmad**, Akshat Chowdhury***
}

\section{ABSTRACT:}

The current study is about thought disorder and its kinds. Disorder of thought refers to the disturbance in one's thoughts. There are many types of thought disorders. Broadly, thought disorders are classified into four main categories: stream, possession, content and form. The study is regarding these four main categories as well as the other types of disorders of thought that may be regarded as their sub-categories. The authors have tried hard enough to explain each type of disorder of thought with the help of an example, making the comprehension easy especially for those who don't know much about thought disorders. Furthermore, the authors have tried to illustrate different types of thought disorders using the illustrative pictures. The authors have gathered information through available manuscripts as well as internet.

Keywords: Disorders, Thought, Stream, Possession

\section{INTRODUCTION}

Personality is the superstructure of the relatively consistent and enduring innate learned, apparent hidden characteristics of an individual like physical appearance, attitudes and behaviour that combine together and influence one's way of interaction with the environment (Ahmad, 2012). Differences of thought also influence personality and that makes difference of one personality to another personality. Disorder of thought refers to the disturbance in the processing, organization and relevance of thoughts manifested as illogical, bizarre, unorganized and irrelevant language or communication. Frank Fish, the first professor for Psychiatry at the University of Liverpool, in 1967, classified disorder of thought into: stream, possession, content and form. Disorder of Stream has two main categories: (1) Disorder of Tempo and (2) Disorder of Continuity. Disorder of Tempo is further divide into: (i) Flight of Ideas (ii) Retardation of Thinking and (iii) Circumstantialities. Disorder of Continuity is divided into: (i) Perseveration and (ii) Thought Blocking. Disorder of Possession has two main categories: (1) Obsession and Compulsion and (2) Thought Alienation. Thought Alienation is further divided into: (i) Thought Insertion (ii) Thought Withdrawal (iii) Thought Broadcasting and (iv) Thought Echo. Disorder of Content is categorized into: (1) Phobias (2) Religious Preoccupations (3) Ideas of Hopelessness

*Assistant Professor and Clinical Psychologist, Dept. of Psychology, AIBAS, Amity University Rajasthan, Jaipur. India $\mid * * \& * * *$ M.Phil Clinical Psychology trainee, Dept. of Psychology, AIBAS, Amity University Rajasthan, Jaipur.

(C) 2014 K Singh; licensee IJIP. This is an Open Access Research distributed under the terms of the Creative Commons Attribution License (http://creativecommons.org/licenses/by/2.0), which permits unrestricted use, distribution, and reproduction in any Medium, provided the original work is properly cited. 
(4) Ideas of Worthlessness (5) Suicidal Ideas (6) Hypochondriacally Ideas (7) Depersonalization (8) Serialization (9) Antisocial Urges (10) Homicidal Ideas and (11) Delusions. Karl Jaspers categorized delusions as: (i) Primary Delusions and (ii) Secondary Delusions. Primary delusions include: (a) Delusional Intuition (b) Delusional Perception (c) Delusional Atmosphere and (c) Delusional Memory. Secondary delusions include: (a) Delusion of Control (b) Nihilistic Delusion (c) Delusional Jealousy (d) Delusion of Guilt (e) Delusion of Mind being Read (f) Delusion of Thought Insertion (g) Delusion of Reference (h) Erotomania (i) Religious Delusion (j) Somatic Delusion (k) Delusion of Poverty (l) Delusion of Persecution (m) Delusion of Grandeur (n) Delusion of Infidelity (o) Delusion of Recognition and (p) Delusional Misidentification Syndrome. Delusional misidentification syndrome further comprises Capgras Delusion, Fregoli Delusion, Intermetamorphosis and Subjective Doubles. Disorder of Form consists of: (1) Loosening of Associations (2) Derailment (3) Neologisms (4) Desultory Thoughts (5) Drivelling (6) Omission (7) Clang Associations and (8) Word Salad.

\section{CONTENT:}

\section{Disorder of Thought:}

Disorder of thought refers to any disturbance in the processing, organization and relevance of thoughts manifested as illogical, bizarre, unorganized and irrelevant language or communication. Thought disorder is the hallmark feature of many psychological disorders especially the psychotic disorders like schizophrenia. Manifestation of thought disorder ranges from simple blocking and mild circumstantialities to profound loosening of association, incoherence and delusion, characterized by a failure to follow semantic and syntactic rules that is inconsistent with the person's education, intelligence or cultural background.

People who suffer from thought disorders often have difficulty with the flow, control, subject matter and organization of thoughts. They may be unable to express thoughts in a logical fashion, or they may speak quickly or incoherently. Many individuals also suffer from a disturbance in the way they produce content- this can be seen in individuals having delusions or hallucinations, such as people suffering from PTSD.

Fish (2007) classified disorder of thought into the following categories:

I. Disorder of Stream of Thought: Abnormality in the flow of one's thoughts.

II. Disorder of Possession of Thought: Loss of control over one's thought processing.

III. Disorder of Content of Thought: Abnormality in content or material of thought.

IV. Disorder of Form of thought: Disturbance in the arrangement of thoughts or in the logical connections between ideas.

Let us elaborate this classification of thought disorders in the following manner: 


\section{DISORDER OF STREAM OF THOUGHT:}

A normal individual has a smooth and continuous flow of thoughts and at the same time he is still able to maintain a tolerable standard in the pace or speed of the flowing thoughts. However, a patient with a disorder of stream of thought finds it very difficult to manage and maintain generally acceptable speed and continuity in his thoughts. Patients with mania, depression and schizophrenia generally possess the disorder of stream of thought. Disorder of stream of thought is of two main types:

- Disorder of Tempo

- Disorder of Continuity

Let us describe them in the following manner:

\section{DISORDER OF TEMPO:}

It refers to the abnormality in the speed of the production of one's thoughts. It can be categorised into the following:

\section{a) Flight of Ideas:}

A nearly continuous flow of rapid speech that jumps from topic to topic, usually based on discernible associations, distractions, or plays on words, but in severe cases so rapid as to be disorganized and incoherent. It is mostly seen in mania, schizophrenia and ADHD. For example, the patient says, "I like oranges, the water is very cool, America rules the world".

\section{b) Inhibition or Retardation of Thinking:}

It refers to the slow speed of speech and prolonged latent period before response. For example, the patient says, "I went to the market (pause)................. the shop was closed." It is mostly seen in schizophrenia, depression.

\section{c) Circumstantialities:}

It occurs when thinking proceeds slowly with many unnecessary and trivial details, but finally the point is reached. For example, when the patient is asked about the bruise on her arm, the patient recounts everything else that happened the same day before explaining how she was injured. It is mostly seen in mania, schizophrenia, organic mental disorders.

\section{DISORDER OF CONTINUITY:}

It refers to the abnormality in the maintenance of a particular matter of concern while thinking about something. It can be categorized into the following types: 


\section{a) Perseveration:}

It refers to a disturbance in thought association that is manifested by repetitive verbalisation or persistent repetition of the same ideas in response to different questions. For example, when asked for the current day, the patient says it's Wednesday, but subsequent questions about month, year, and place are all met with the same reply. It is mostly seen in schizophrenia, dementia, etc.

\section{b) Thought blocking:}

It refers to the abrupt interruption in the flow of thoughts or ideas resulting from a disturbance in the speed of association. For example, while responding to the query about how he was injured, the patient says, "I went to the market (extreme silence)..............." This disorder is mostly seen in schizophrenia, anxiety disorder, etc.

\section{DISORDER OF POSSESSION OF THOUGHT:}

Normally, one experiences one's thinking as being one's own, although this sense of personal possession is never in the foreground of one's consciousness. One also feels that one is in the control of one's thinking. However, in some psychiatric illnesses, there is a loss of control of sense of possession of thinking. Disorder of possession of thought can be divided into the following categories: (Casey \& Kelley, 2007)

- Obsession and Compulsion

- Thought Alienation

These can be described in the following manner:

1. OBSESSION AND COMPULSION (Obsessive Compulsive Disorder, 2014):

It is important to understand the distinction between obsessions and compulsions. Obsessions are recurrent and persistent thoughts, impulses, or images that cause distressing emotions such as anxiety or disgust despite the individual's awareness that the thought is either entirely without purpose or else has persisted and dominated their thinking beyond the point of relevance or usefulness. People with OCD recognize that the thoughts, impulses, or images are a product of their mind and are excessive or unreasonable. Yet these intrusive thoughts cannot be settled by logic or reasoning.

One of the most important characteristic features of obsession is that their content causes the sufferer great anxiety and even guilt. The thoughts are particularly repugnant (disgusting) to the individual like for example; the prudish person is tormented by sexual thoughts, the religious person by blasphemous thoughts and the timid person by thoughts of torture, murder and general mayhem. Generally, nowadays, the most common forms of obsession tend to be concerned with 


\section{Differences of thought}

fears of doing harm e.g., wife with an obsession that she may harm her husband. Most people try to ignore or suppress such obsessions or neutralize them with some other thought or action.

Some typical obsessions include:

(a) Fear of dirt or contamination, (b) Fear of becoming ill, (c) Constant thoughts of a certain number, (d) Need for symmetry or exactness, (e) Worry about whether something has been done "right", (f) Forbidden sexual or religious thoughts

While obsessions are recurring thoughts, compulsions are recurrent actions. Compulsions are repetitive behaviours or actions that the person feels driven to perform in response to an obsession. The behaviours are aimed at preventing or reducing distress or a feared situation. In the most severe cases, a constant repetition of rituals may fill the day, making a normal routine impossible. For example, a person may need to check to see if they locked the doors before going to bed. They may lose sleep continuing to check over and over. Mostly these will be quite mild but in more extreme cases they may develop as a means for people to try to relieve themselves from their obsessive thoughts. For example, when there is an obsession with germs, a person may continually wash their hands, even to the point their hands become raw from so much washing. When a person continues to repeat an action, obsessions may go away for a short period of time, however, they will normally return. Once they return, the compulsion will begin again, starting a cycle of obsession-compulsion.

Some common compulsions include:

(a) Washing hands, (b) Brushing teeth, (c) Blinking eyes , (d) Counting items, such as currency, (f) Checking to see if appliances are turned off, (g) Checking to see if doors are locked, (h) Arranging items in a certain way, (i) Keeping items, such as containers, even if they are no longer needed, (j) Requiring constant approval from people around them.

Obsession occurs in obsession states, depression, schizophrenia, and occasionally in organic states; compulsive features appear to be particularly common in post-encephalitic Parkinsonism (Lishman, 1998).

\section{THOUGHT ALIENATION: (Cassey and Kelley, 2007)}

This refers to a symptom of psychosis in which the patients have the experience that their thoughts are under the control of an outside agency or that others are participating in their thinking. Any form of thought alienation is a highly indicative feature of schizophrenia. Thought alienation includes the following categories:

\section{(a) Thought Insertion:}

In pure thought insertion, the patient knows that thoughts are being inserted into their mind and they recognize them as being foreign and coming from without any stimulus. This symptom is commonly associated with schizophrenia, is not unique to schizophrenia and a range of related 
phenomena have also been described. For example, the patient repeatedly complains of having disturbed violent thoughts, which, she claims are being sent to her by alien.

\section{(b) Thought Withdrawal:}

Thought withdrawal refers to the delusion that someone or something is removing thoughts from one's mind and the patient has no power over this. For example, the patient continually blames his poor memory on "government agents" who he claims are able to steal his thoughts.

\section{(c) Thought Broadcasting:}

Here the patient knows that as they are thinking, everyone else is thinking in unison with them (Fish \& Hamilton, 1994). The term "Thought Broadcasting" has also been used to describe the belief that one's thoughts are quietly escaping from one's mind and that other people might be able to access them, and the experience of hearing one's thoughts spoken aloud and believing that, as a result, other people can hear them. For example, the patient believes that the news broadcasted on television or internet actually reflects his own thoughts.

\section{(d) Thought Echo:}

In this type of thought disorder, the patient hears the 'echo' of his own thoughts in the form of a voice after he has made the thought. For example, the patient may complain that when he thought of meeting a person in the morning, he heard a voice uttering the same thought aloud.

\section{DISORDER OF CONTENT OF THOUGHT:}

Normal contents of thought are composed of awareness, ideas, concern, belief, preoccupation, imagination (The Phobia List, 1995).

- Phobia, • Religious Preoccupations • Ideas of Hopelessness • Ideas of Worthlessness • Suicidal

Ideas - Hypochondriacally Ideas - Depersonalization - Serialization • Antisocial Ideas • Homicidal Ideas • Sex Preoccupations • Delusions

Now, let us describe these abnormalities in the following manner:

\section{PHOBIAS:}

A phobia is a type of anxiety disorder, usually defined as a persistent fear of an object or situation in which the sufferer commits to great lengths in avoiding, typically disproportional to the actual danger posed, often being recognized as irrational. In the event the phobia cannot be avoided entirely, the sufferer will endure the situation or object with marked distress and significant interference in social or occupational activities. 
Some of the common phobias are:

1. Arachnophobia - fear of spider

2. Barophobia - fear of gravity

3. Cynophobia - fear of dogs

4. Demonophobia, - fear of demons

5. Emetophobia - fear of vomiting

6. Frigophobia - fear of becoming too cold

7. Gephyrophobia - fear of bridges

8. Hemophobia - fear of blood

9. Ichthyophobia - fear of fish

10. Kinetophobia - fear of movement

11. Logophobia - fear of words

12. Melissophobia - fear of bees

13. Nosocomephobia - fear of hospitals

14. Ombrophobia - fear of rain

15. Pyrophobia - fear of fire

16. $\cdot$ Radiophobia - fear of radioactivity or X-rays

17. Scolionophobia - fear of school

18. Thanatophobia - fear of dying

19. Uranophobia - fear of Heaven

20. Vestiphobia - fear of clothing

21. Workplace phobia - fear of the workplace

22. Xanthophobia - fear of the color yellow

23. Zoophobia - fear of animals

\section{RELIGIOUS PREOCCUPATIONS:}

This refers to the thoughts involving religious themes or subject matter. Individuals experiencing religious preoccupations are anxious with religious subjects that are not within the expected beliefs for an individual's background, including culture, education and known experiences of religion. These preoccupations are incongruous with the mood of the subject. For example, the patient may believe that he gets instructions directly from God. These are found in patients with schizophrenia and psychotic depression.

\section{IDEAS OF HOPELESSNESS:}

This refers to the thoughts of complete despair where the patient strongly believes that the circumstances will never get better for him. For example, the patient may think that the government will never be able to remove corruption from the society. Such thoughts are commonly found in patients with Generalised Anxiety Disorder and Depression. 


\section{IDEAS OF WORTHLESSNESS:}

When someone feels worthless, they feel as if they are insignificant and have nothing valuable to offer the world. They feel as if their entire life is cast in a negative light with no prospect of improvement. Often, this perception is extremely distorted and is the result of an underlying condition such as depression, anxiety, grief, or stress. For example, the patient says that his life is a complete waste.

\section{SUICIDAL IDEAS:}

It refers to the thoughts of taking one's own life. Generally, the patients with the thoughts of hopelessness and worthlessness suffer from the thoughts of suicide as well.

\section{HYPOCHONDRIACAL IDEAS:}

Patients having these ideas become unduly alarmed about any physical or psychological symptoms they detect, no matter how minor the symptom may be and are convinced that they have or are about to be diagnosed with, a serious illness. For example, the patient may be very curious to gather information about different serious diseases and try to correlate his signs with the symptoms.

\section{DEPERSONALISATION:}

Here, the sufferers feel disconnected from one's physicality or body, feeling detached from one's own thoughts or emotions, feeling as if one is disconnected from reality and a sense of feeling as if one is dreaming or in a dreamlike state.

\section{DEREALISATION:}

Derealisation is an alteration in the perception or experience of the external world so that it seems unreal. Other symptoms include feeling as though one's environment is lacking in spontaneity, emotional colouring and depth. It is a dissociative symptom of many conditions such as psychiatric and neurological disorders and not a standalone disorder. For example, the patient may complain that the world and the aspects of it are nothing more than a dream.

\section{ANTISOCIAL URGES:}

This refers to the thoughts of indulging in some behaviours or actions that harm or lack consideration for the well-being of others. Anti-social behaviour is labelled as such when it is deemed contrary to prevailing norms for social conduct. For example, the patient may feel highly motivated to rob a bank or to threaten people for extortion money.

\section{HOMICIDAL IDEAS:}

Here, the patient has a range of homicidal thoughts which spans from ideas of revenge to detailed and fully formulated plans without the act itself. 


\section{Differences of thought}

\section{SEX PREOCCUPATION:}

Here, the patient is preoccupied with thoughts of sexual acts or behaviours.

\section{DELUSIONS:}

Delusions are false beliefs that are strongly held and unchangeable in the face of refuting evidence and that are not consistent with the person's educational, social and cultural background.

The psychiatrist and philosopher Jaspers (1913) define the three main criteria for a belief to be considered delusional in his book General Psychopathology. These criteria are:

i. Certainty (held with complete confidence)

ii. Incorrigibility (not changeable by proof to the contrary)

iii. Impossibility or falsity of content (implausible, bizarre or patently untrue)

Delusions are categorized into the following groups:

i) Bizarre Delusion: A delusion that is very strange and completely implausible. For example, the patient may believe that aliens have removed his brain or heart.

ii) Non-bizarre Delusion: A delusion that, though false, is at least possible. For example, the patient may believe that he is under constant police surveillance.

iii) Mood-congruent Delusion: Any delusion with content consistent with either a depressive or manic state. For example, the patient with depression may believe that news anchors on television highly disapprove of him.

iv) Mood-incongruent Delusion: A delusion that does not relate to the sufferer's emotional state; for example, a belief that an extra limb is growing out of the back of one's head is incongruent to either depression or mania.

v) Simple Delusion: Contains relatively few elements.

vi) Complex Delusion: Contains extensive elaboration of people, spirits, motives and situation.

vii) Systematised Delusion: Systematised delusions are restricted to well delineate areas and associated with clear sensorium.

viii) Non-systematised Delusion: Delusions that are extended up to many aspects of life, new data, people and situation. 
ix) Complete Delusion: Complete delusions are held utterly without any doubt.

x) Partial Delusion: In partial delusion, the patient entertains doubt about the delusional idea or belief.

xi) Primary Delusion: Primary delusions are those that are not understandable in terms of patient's culture or mood also called autochthonous delusion. It occurs in an instant without identifiable preceding event.

xii) Secondary Delusion: Secondary delusions are those that are atleast understandable in terms of the patient's culture or mood.

Jaspers originally made a distinction between primary and secondary delusions. Let us understand the difference through explanations in the following manner:

\section{PRIMARY DELUSION:}

According to Karl Jaspers, primary delusions (sometimes called true delusions) are distinguished by a transformation of meaning, so that the world or aspects of it are interpreted in a radically different way by the delusional person. To others, this interpretation is 'un-understandable' in terms of the normal mental causality, mood, environmental influences and other psychological or psychopathological factors. Jaspers describes four types of primary delusion:

a) Delusional Intuition - where delusions arrive 'out of the blue', without external cause.

b) Delusional Perception - where a normal percept is interpreted with delusional meaning. For example, a person sees a red car and knows that this means their food is being poisoned by the police.

c) Delusional Atmosphere - where the world seems subtly altered, uncanny, portentous or sinister. This resolves into a delusion, usually in a revelatory fashion, which seems to explain the unusual feeling of anticipation.

d) Delusional Memory - where a delusional belief is based upon the recall of memory or false memory for a past experience. For example, a man recalls seeing a woman laughing at the bus stop several weeks ago and now realises that this person was laughing because the man has animals living inside him.

\section{SECONDARY DELUSIONS:}

Secondary delusions (sometimes called delusion-like ideas) are considered to be, at least in principle, understandable in the context of a person's life history, personality, mood state or presence of other psychopathology. For example, a person becomes depressed, suffers very low mood and self-esteem, and subsequently believes they are responsible for some terrible crime which they did not commit. 
Delusions often manifest according to a consistent theme. Although delusions can have any theme, certain themes are more common. Some of the more common delusion themes are:

\section{a) Delusion of Control:}

This is a false belief that another person, group of people, or external force controls one's general thoughts, feelings, impulses, or behaviour.

\section{b) Nihilistic Delusion or Cotard Delusion:}

This is a false belief that one does not exist, has died or has lost a body organ. It was first described by Dr. Jules Cotard, a French neurologist, in 1880.

\section{c) Delusional Jealousy:}

A person with this delusion falsely believes that a spouse or lover is having an affair, with no proof to back up their claim.

\section{d) Delusion of Guilt or Sin (or delusion of self-accusation):}

This is an ungrounded feeling of remorse or guilt of delusional intensity. For example, the patient may believe that he has done something wrong for which he has to undergo the punishment.

\section{e) Delusion of Mind being Read:}

It refers to the false belief that other people can know one's thoughts. This is different from thought broadcasting in that the person doesn't believe that his or her thoughts are heard aloud.

\section{f) Delusion of Thought Insertion:}

It refers to the belief that another thinks through the mind of the person. The person may sometimes be unable to distinguish between their own thoughts and those inserted into their minds. A person with this delusional belief is found to be convinced of their beliefs and unwilling to accept such diagnosis.

\section{g) Delusion of Reference:}

Here, the person falsely believes that insignificant remarks, events or objects in one's environment have personal meaning or significance. For example, a person with schizophrenia might believe a billboard or a celebrity is sending a message meant specifically for them.

\section{h) Erotomania:}

It refers to a delusion in which a person falsely believes that a particular individual, usually a celebrity or someone of a higher status is in love with him/her. Such a person usually attempts to contact the other person through phone calls, letters or gifts. 


\section{i) Religious Delusion:}

A religious delusion is any delusion involving religious or spiritual themes or subject matter. For example, a person may believe that he is a god, or given the authority to act as a god.

\section{j) Somatic Delusion:}

It refers to a delusion whose content pertains to bodily functioning, bodily sensations, or physical appearance. Usually the false belief is that the body is somehow diseased, abnormal, or changed. A specific example of this delusion is delusional parasitosis: a delusion in which one feels infested with insects, bacteria, mites, spiders, lice, fleas, worms or other organisms.

\section{k) Delusion of Poverty:}

Here, the person strongly believes that he is financially incapacitated. This type of delusion is less common now.

\section{l) Delusion of Persecution:}

Here, the patient believes that others - often a vague "they" — are out to get him or her. These persecutory delusions often involve bizarre ideas and plots. For example, one believes that Russians are trying to poison him with radioactive particles delivered through his tap water.

\section{m) Delusion of Grandeur:}

It refers to the belief that one is a famous or important figure, such as William Shakespeare or Abraham Lincoln. Alternately, delusions of grandeur may involve the belief that one has unusual powers that no one else has e.g. the ability to fly.

\section{n) Delusion of Infidelity:}

Here, the patient holds a strong delusional belief that their spouse or sexual partner is being unfaithful to him or her without having any significant proof to back up their claim.

\section{o) Delusion of Recognition:}

This refers to the belief that an event or incident that is being experienced currently has already been experienced in the past. Neppe (1983) described 20 different types of such experiences in his book titled The Psychology of Déjà Vu. Some of these have specific names:

- déjà vécu already lived through or experienced

- déjà senti already felt

- déjà visite already visited

- déjà entendu already heard 
- déjà éprouvé already tried or attempted

- déjà fait already done or accomplished

• déjà pensé already thought or pondered

- déjà raconté already recounted or told

- déjà su already known (intellectually)

- déjà trouvé already met

• déjà voulu already wanted"

\section{o) Delusional Misidentification Syndrome:}

Delusional misidentification syndrome is an umbrella term, introduced by Christodoulou (1986) for a group of delusional disorders that occur in the context of mental or neurological illness. They all involve a belief that the identity of a person, object or place has somehow changed or has been altered. As these delusions typically only concern one particular topic they also fall under the category called monothematic delusions.

This syndrome is usually considered to include four main variants:

i. The Capgras Delusion: It is the belief that (usually) a close relative or spouse has been replaced by an identical-looking impostor.

ii. The Fregoli Delusion: It is the belief that various people the believer meets are actually the same person in disguise.

iii. Intermetamorphosis: It is the belief that people in the environment swap identities with each other whilst maintaining the same appearance.

iv. Subjective Doubles: Subjective doubles is the belief that there is a doppelgänger or double of him or herself carrying out independent actions.

However, similar delusional beliefs, often singularly or more rarely reported, are sometimes also considered to be part of the delusional misidentification syndrome. For example:

i. Mirrored-self Misidentification: It is the belief that one's reflection in a mirror is some other person.

ii. Reduplicative Paramnesia: It is the belief that a familiar person, place, object or body part has been duplicated. For example, a person may believe that they are in fact not in the hospital to which they were admitted, but an identical-looking hospital in a different part of the country, despite this being obviously false. 
iii. Syndrome of Delusional Companions: It is the belief that objects (such as soft toys) are sentient beings.

iv. Clonal Pluralization of the Self: It is where a person believes there are multiple copies of him or herself, identical both physically and psychologically but physically separate and distinct.

There is considerable evidence that disorders such as the Capgras or Fregoli syndromes are associated with disorders of face perception and recognition. However, it has been suggested that all misidentification problems may exist on a continuum of anomalies of familiarity, from déjà $\mathrm{vu}$ at one end to the formation of delusional beliefs at the other.

\section{DISORDER OF FORM OF THOUGHT:}

Disorder of form of thought is also called formal thought disorder. This is disorder of conceptual or abstract thinking. Disorders of the form of thought is usually recognised from speech and writing but is sometimes evident from the patient's behaviour e.g., he may be unable to file papers under appropriate category headings.

The form of thought is largely assessed by examining the speech of the patient. Thought is also reflected in behaviour but behaviour is also dependent on factors like personality, motivation and other cognitive processes like memory and orientation. Formal thought disorder typically refers to marked abnormality in the form and flow of thought.

Disorders of form of thought can be divided into three main kinds as under:

- Loosening of Associations

- Derailment

- Neologisms

- Desultory Thoughts

- Drivelling

- Omission

- Clang Associations

- Word salad

These can be described in the following manner:

\section{LOOSENING OF ASSOCIATIONS:}

This denotes a loss of the normal structure of thinking. To the interviewer, the patient's discourse seems muddled, illogical or tangential to the matter in hand. It does not become clearer when the patient is questioned further, indeed the interviewer has the experience that the more he tries to clearly the patient's thinking, the less he understands it. Several specific features of this muddled thinking have been described, but they are difficult to identify with certainty and the most striking clinical impression is often a general lack of clarity. 


\section{DERAILMENT:}

It is characterized by a speech consisting of a sequence of unrelated or only remotely related ideas. In a mild manifestation, this thought disorder is characterized by slippage of ideas further and further from the point of a discussion. For example, "Some people just don't understand why I had breakfast in America during the world war.

\section{NEOLOGISMS:}

A neologism refers to a newly coined term, word, or phrase created by combining different words or by breaking them. The use of neologisms is common in children, but is considered indicative of a thought disorder when present in adults. For example, the patient says, "The world is not gooder for people like me!" combining two words: "GOOD and BETTER".

\section{DESULTORY THOUGHTS:}

Here, the speech is grammatically correct however, sudden unrelated ideas force their way in from time to time. The unconscious part of one's mind may be forcing the patient to include other information in his conscious speech. For example, the patient says "My actual problem is that the people never understand me and my stomach feels like an empty bucket" by which he actually means that he is hungry.

\section{DRIVELLING:}

This refers to mixing of thoughts which makes comprehension difficult. Here, the listener initially feels that the patient has a good idea about what he is going to say further but then he suddenly loses preliminary organization of the thought so that all the constituents get muddled together. For example, the patient says, "Yes, actually that person created all the trouble in my life, ... the weather is getting colder day by day".

\section{OMISSION:}

It refers to the exclusion of some relevant information by the patient while verbally responding to a query. Sometimes, an omitted portion of information may be highly suggestive of the cause of the problems faced by the patient. For example, while giving a detailed account of his family members, the patient may consciously or unconsciously skip the information.

\section{CLANG ASSOCIATIONS:}

Clang associations refer to a mode of speech characterized by association of words based upon sound or tunes of the words rather than concepts or meanings. This is associated with the irregular thinking apparent in psychotic disorders like schizophrenia. For example, the patient says, "I heard the bell, well, hell, then I fell." 


\section{WORD SALAD:}

Word salad is a confused or unintelligible mixture of seemingly random words and phrases. The words may or may not be grammatically correct, but semantically confused to the point that the listener cannot extract any meaning from them. For example, the patient says, "Tree bus rain food home car tiger".

\section{CONCLUSION:}

In this study, the authors have tried their best to provide a detailed view of the disorders of thought by categorising and classifying the different types and subtypes of these disorders. However, keeping the vast scope of the area under study in mind, the authors wholeheartedly accept that there may be many more types of thought disorders that may have skipped the required attention. Therefore, further study is warranted so that more light will be thrown on the dark corners of this area. Let us conclude with some words of wisdom by Allan Lokos, he rightly says, "Don't believe everything you think......"

\section{REFERENCES:}

1. Ahmad, A. (2012). Exploring the relationship between Spiritual Personality and Emotional Empathy among Medical and Unani Students. (Unpublished Dissertation). Aligarh Muslim University, India: AL.

2. Casey, P., \& Kelley, B. (2007). Fish's Clinical Psychopathology: Signs and symptoms in Psychiatry ( $3^{\text {rd }}$ Edu.). Jaypee brothers Medical Publishers (P) Ltd. India: New Delhi.

3. Christodoulou, G.N. (1986). The Delusional misidentification syndromes. Basel;Munchen; Paris : Karger.

4. Fish, F. J., Patricia R. C. \& Kelly, B. (2007). Fish's Clinical Psychopathology: Signs and Symptoms in Psychiatry. London : The Royal College of Psychiatrists.

5. Fish, F.J., \& Hamilton, M. (1994). Fish's Clinical Psychopathology, Bombay: Varghese Publishing House.

6. Jaspers, K. (2008). Allgemeine Psychopathologie. Ein Leitfaden für Studierende, Arzte und Psychologen. Berlin: J. Springer.

7. Lishman, W.A. (1998). Organic psychiatry: The Psychological consequences of cerebral Disorder $\left(3^{\text {rd }}\right.$ Edu). Oxford: Black Well Science.

8. Neppe, V.M. (1983). The psychology of déjà vu: have I been here before? Witwatersrand University Press: the University of Michigan.

9. Obsessive Compulsive Disorder (2014) American Psychiatric Association. Retrieved from http://www.psychiatry.org/obsessive-compulsive-disorder on 14/9/2014

10. The Phobia List (1995). Retrieved from http://www.phobialist.com on 17/9/2014. 